\title{
ON THE EXISTENCE OF VARIATIONS, POSSIBLY WITH POINTWISE GRADIENT CONSTRAINTS
}

\author{
Simone Bertone ${ }^{1}$ And Arrigo Cellina ${ }^{1}$
}

\begin{abstract}
We propose a necessary and sufficient condition about the existence of variations, i.e., of non trivial solutions $\eta \in W_{0}^{1, \infty}(\Omega)$ to the differential inclusion $\nabla \eta(x) \in-\nabla u(x)+\mathbf{D}$.
\end{abstract}

Mathematics Subject Classification. 49K10, 35F30.

Received February 16, 2006.

\section{A CONJECTURE}

Purpose of the present paper is to derive conditions for the existence of (non trivial) solutions $\eta \in W_{0}^{1, \infty}(\Omega)$ to the differential inclusion

$$
\nabla \eta(x) \in-\nabla u(x)+\mathbf{D}
$$

where $\mathbf{D}$ is a given set and $u$ is in $W^{1,1}(\Omega)$ and satisfies

$$
\nabla u(x) \in \operatorname{co}(\mathbf{D})
$$

(in the case $\mathbf{D}$ is convex, $\eta=0$ is always a solution).

The problem of characterizing conditions for the existence of solutions is complex: in $\mathbb{R}^{2}$, consider the function $v\left(x_{1} ; x_{2}\right)=\sqrt{x_{1}^{2}+x_{2}^{2}}$ whose gradient satisfies $\|\nabla v(\cdot)\|=1$, let $\mathbf{B}$ be the unit ball of $\mathbb{R}^{2}$ and, on $\Omega \subset \mathbb{R}^{2}$, consider the inclusion

$$
\nabla \eta \in-\nabla v+\mathbf{B} .
$$

When $\Omega$ is the open disk $x_{1}^{2}+x_{2}^{2}<R^{2}$, it is easy to see that non trivial solutions $\eta$ do exist; however, when $\Omega$ is the annulus $r^{2}<x_{1}^{2}+x_{2}^{2}<R^{2}$, nontrivial solutions do not exist. Hence, the existence or non-existence of nontrivial solutions depends on the geometry of $\Omega$, and cannot be expressed by local conditions.

As a motivation for the problem, and for the name of variations proposed here for the solutions $\eta$, assume we are considering the problem of minimizing

$$
\int_{\Omega} L(\nabla v(x)) \mathrm{d} x
$$

Keywords and phrases. Variations, differential inclusions, necessary conditions.

1 Dipartimento di Matematica e Applicazioni, Universitá degli Studi di Milano-Bicocca, Via R. Cozzi 53, 20125 Milano, Italy; simone.bertone@unimib.it; arrigo.cellina@unimib.it 
under given boundary conditions, where $L$ is a convex function, for instance

$$
L(\xi)=\left\{\begin{array}{cc}
1-\sqrt{1-\|\xi\|^{2}} & \text { for }\|\xi\| \leq 1 \\
+\infty & \text { elsewhere }
\end{array}\right.
$$

$L$ is finite for $\xi$ in $\mathbf{B}$, the unit ball of $\mathbb{R}^{N}$ equipped with the Euclidean norm. Let $u$ be a solution to the minimum problem, and assume that we wish to derive the necessary conditions satisfied by $u$, hence to compare the values of the integral functional at $u$ and at $u+\eta$. To find these conditions, we have to ask ourselves whether there are nontrivial variations $\eta$, such that $\|\nabla u(x)+\nabla \eta(x)\| \leq 1$, i.e., solutions to $\nabla \eta(x) \in-\nabla u(x)+\mathbf{B}$. In this case the function $u$, appearing in the differential inclusion we are investigating, is interpreted as the solution to a variational problem and the set $\mathbf{D}$ as the effective domain of a convex Lagrangean.

We propose the following conjecture, on the existence of non trivial variations. In it, and in the remainder of the paper, by saying that a vector function $p \in L_{l o c}^{1}(\Omega)$ is such that $\operatorname{div}(p)=0$ we mean that, for every $\eta \in C_{c}^{\infty}(\Omega)$, we have

$$
\int_{\Omega}\langle p(x), \nabla \eta(x)\rangle \mathrm{d} x=0 .
$$

Conjecture. Let $\mathbf{D} \subset \mathbb{R}^{N}$. Let $u$ be a solution to

$$
\nabla u(x) \in \operatorname{co}(\mathbf{D}) .
$$

Then, the following a) and b) are in alternative:

a) there exists a nontrivial $\eta \in W_{0}^{1, \infty}(\Omega)$, solution to

$$
\nabla \eta(x) \in-\nabla u(x)+\mathbf{D}
$$

b) there exists a vector function $p \in L_{\text {loc }}^{1}(\Omega), p(x) \neq 0$ a.e., such that $\operatorname{div}(p)=0$, and

$$
\langle p(x), \nabla u(x)\rangle=\sup _{k \in \mathbf{D}}\langle p(x), k\rangle
$$

for almost every $x \in \Omega$.

\section{Examples.}

1) In the case $\mathbf{D}=\mathbb{R}^{N}$, condition b) is never satisfied and variations do always exist.

2) Consider again the function $v\left(x_{1} ; x_{2}\right)=\sqrt{x_{1}^{2}+x_{2}^{2}}=\rho$, whose gradient $\nabla v\left(x_{1} ; x_{2}\right)=\frac{1}{\rho}\left(x_{1} ; x_{2}\right)$. When $\Omega$ is the annulus $r^{2}<x_{1}^{2}+x_{2}^{2}<R^{2}$, non trivial solutions do not exist, hence a) is never satisfied. Let us show that $\mathrm{b})$ is true: the vector function $p\left(x_{1} ; x_{2}\right)=\frac{1}{x_{1}^{2}+x_{2}^{2}}\left(x_{1} ; x_{2}\right)$ has pointwise divergence zero everywhere in $\Omega$; moreover

$$
\sup _{k \in B}\left\langle p\left(x_{1} ; x_{2}\right), k\right\rangle=\frac{1}{\rho}=\left\langle p\left(x_{1} ; x_{2}\right), \nabla v\left(x_{1} ; x_{2}\right)\right\rangle .
$$

Hence b) is satisfied.

When $\Omega$ is the open disk $x_{1}^{2}+x_{2}^{2}<R^{2}$, non trivial $\eta$ exist, so a) is satisfied. The vector $p$ as used before has not weak divergence zero in $\Omega$, hence it does not prove that b) is satisfied. The fact that b) cannot be satisfied will be proved below.

In the present paper we prove the above conjecture under some additional regularity assumption. 


\section{The CASe $\nabla u=0$}

In this section we show that the Conjecture is verified in the case $\nabla u=0$.

Theorem 1. Let $\mathbf{D} \subset \mathbb{R}^{N}$ and let $u$ be such that $\nabla u=0 \in \operatorname{co}(\mathbf{D})$. Then, the following a) and b) are in alternative:

a) there exists a nontrivial $\eta \in W_{0}^{1, \infty}(\Omega)$, solution to $\nabla \eta(x) \in \mathbf{D}$;

b) there exists a vector function $p \in L_{\text {loc }}^{1}(\Omega), p(x) \neq 0$ a.e., such that $\operatorname{div}(p)=0$, and for a.e. $x \in \Omega$,

$$
\sup _{k \in \mathbf{D}}\langle p(x), k\rangle=0 .
$$

In the proof of Theorem 1 we will need the following lemma, whose proof is a consequence of a result appearing in $[4]$.

Lemma 1. Let $\Omega \subset \mathbb{R}^{N}$ an open bounded set, and $\mathbf{D} \subset \mathbb{R}^{N}$. There exists a nontrivial function $\eta \in W_{0}^{1, \infty}(\Omega)$ such that $\nabla \eta(x) \in \mathbf{D}$ for a.e. $x \in \Omega$, if and only if $0 \in \operatorname{int}(\operatorname{co}(\mathbf{D}))$.

Proof. When $0 \in \operatorname{int}(\operatorname{co}(\mathbf{D}))$, by Lemma 1 , there exists $\eta \in W_{0}^{1, \infty}(\Omega)$ such that, a.e., $\nabla \eta(x) \neq 0$, hence $\eta$ is non trivial and a) is always satisfied. We show that b) cannot be true: in fact, in this case, there must exist a ball $B(0, r) \subset c o(\mathbf{D})$ so that, for every non trivial vector function $p$, we have $\langle p(x), \nabla u(x)\rangle \equiv 0$, while $\sup _{k \in \mathbf{D}}\langle p(x), k\rangle \geq r\|p(x)\|$, that is positive on a set of positive measure.

When $0 \notin \operatorname{int}(\operatorname{co}(\mathbf{D}))$, again by Lemma 1 , there is no $\eta \in W_{0}^{1, \infty}(\Omega)$ apart from $\eta=0$, so that a) is not satisfied. We show that $\mathrm{b}$ ) is true: in fact, the convex sets 0 and $c o(\mathbf{D})$ can be weakly separated, i.e. there exists a non zero vector $v$ such that $\langle v, k\rangle \leq 0$ for every $k \in c o(\mathbf{D})$, i.e., $\operatorname{such}$ that $\sup _{k \in c o(\mathbf{D})}\langle v, k\rangle \leq 0$. This constant vector $v$ is the required $p:$ we have $\sup _{k \in c o(\mathbf{D})}\langle v, k\rangle \leq\langle v, 0\rangle=0$ while, since $0 \in \operatorname{co}(\mathbf{D}), \sup _{k \in c o(\mathbf{D})}\langle p, 0\rangle \geq 0$. This ends the proof.

\section{3. b) imPliES NOT a)}

We prove that b) implies non a) under the additional assumption that $p$ be locally Lipschitzian in $\Omega$, but no special assumptions on $\mathbf{D}$.

Theorem 2. Let $\Omega \subset \mathbb{R}^{N}$ be open, $u \in W^{1,1}(\Omega)$ with $\nabla u(x) \in \operatorname{co}(\mathbf{D})$ for a.e. $x \in \Omega$. Assume that there exists a vector function $p \in W_{\text {loc }}^{1, \infty}(\Omega), p(x) \neq 0$ for $x \in \Omega$, such that $\operatorname{div}(p)=0$ and, for a.e. $x \in \Omega$,

$$
\langle p(x), \nabla u(x)\rangle=\sup _{k \in \mathbf{D}}\langle p(x), k\rangle
$$

Then the only solution $\eta \in W_{0}^{1, \infty}(\Omega)$ to the differential inclusion

$$
\nabla \eta(x) \in-\nabla u(x)+\mathbf{D}
$$

is $\eta \equiv 0$.

In the proof we will need the following lemma, a well known result (Liouville's Theorem) for the case of a differentiable $p$.

Lemma 2. Let $p$ as in Theorem 2. Let $S(t ; x)$ be the solution to the Cauchy problem $\dot{x}(t)=p(x(t)), x(0)=x$. Then the map $x \rightarrow S(t ; x)$ is measure preserving.

Proof of Lemma 2. Let $\widetilde{\Omega} \subset \Omega$ and $\delta>0$ be such that solutions issuing from $\widetilde{\Omega}$ are defined on the interval [0, $\delta]$. We wish to prove that for $t \in[0, \delta]$ and $x \in \widetilde{\Omega}, J(t ; x)$, the Jacobian of the transformation $x \rightarrow S(t ; x)$, equals 
1 a.e. By Rademacher's Theorem, for a.e. $x,(D p)$, the matrix of (pointwise) partial derivatives of $p$ exists. By a result of Tsuji [9], for a.e. $x$,

$$
J(t ; x)=\mathrm{e}^{\int_{0}^{t} \operatorname{tr}((D p)(\tau)) \mathrm{d} \tau}
$$

where the matrix $(D p)$ is computed along the solution $S(\tau ; x)$. We wish to show that for a.e. $x \in \Omega$, for a.e. $t \in[0, \delta]$, we have $\operatorname{tr}((D p)(\tau))=0$. Let $g$ be any of the components of the vector $p$; fix $\eta \in C_{c}^{\infty}(\Omega)$. The sequence $\frac{g\left(x+h_{n} e_{i}\right)-g(x)}{h_{n}}$ converges pointwise a.e. to $\frac{\partial g(x)}{\partial x_{i}}$ and it is (locally) uniformly bounded, so that

$$
\frac{1}{h_{n}} \int\left[g\left(x+h_{n} e_{i}\right)-g(x)\right] \eta(x) \mathrm{d} x
$$

converges both to $\int \frac{\partial g(\xi)}{\partial \xi_{i}} \eta(\xi) \mathrm{d} \xi$ and $\left([6]\right.$, p. 132), to $\int g_{i}(x) \eta(x) \mathrm{d} x$, with $g_{i}$ the $i$ th Sobolev partial derivative of $g$. So $\int\left[\frac{\partial g(x)}{\partial x_{i}}-g_{i}(x)\right] \eta(x) \mathrm{d} x=0$, hence $\frac{\partial g(x)}{\partial x_{i}}-g_{i}(x)=0$, for all components $g$ and all $i$, with the exception of a set $E \subset \Omega$ of $N$ dimensional measure zero. In particular, on $\Omega \backslash E$, the pointwise divergence of $S$ with respect to $x, \operatorname{tr}(D p)$ and the weak divergence $\operatorname{div}(p)$, coincide and are zero.

For $t$ in $[0, \delta]$ and $y$ in $\{S(t ; x): x \in \widetilde{\Omega}\}$ define the inverse map

$$
S^{-1}(t ; y)=(t ; x)
$$

$S^{-1}$ is locally Lipschitzian in its variables and sends the set $[0, \delta] \times E$ into a set $E^{*} \subset([0, \delta] \times \widetilde{\Omega})$ of $N+1$ dimensional measure zero. By Fubini's Theorem, with the exception of a set $X_{E^{*}}$ of $N$ dimensional measure zero, the segments $\{(t ; x): t \in[0, \delta]\}$ meet the set $E^{*}$ on a set of 1 dimensional measure zero. This means that for $x \notin X_{E^{*}}$, for a.e. $t \in[0, \delta], S(t ; x) \notin([0, \delta] \times E)$, i.e., that $\operatorname{tr}(D p(x))$ and $\operatorname{div}(p)$, computed along $S(t ; x)$, coincide.

Proof of Theorem 2.

a) We first notice that condition (2) implies that $\nabla u(x) \in \partial(\operatorname{co}(\mathbf{D}))$ for a.e. $x \in \Omega$.

In fact, otherwise, we can find a set, of positive measure, $\Omega_{*} \subset \Omega$ and $\varepsilon>0$ such that $\nabla u(x)+\varepsilon p(x) \in c o(\mathbf{D})$. For $x \in \Omega_{*}$, we have

$$
\begin{gathered}
\left.\sup _{k \in c o(\mathbf{D})}\langle p(x), k\rangle \geq\langle p(x), \nabla u(x)+\varepsilon p(x)\rangle=\langle p(x), \nabla u(x)\rangle+\varepsilon\|p(x)\|^{2}\right\rangle \\
\langle p(x), \nabla u(x)\rangle=\sup _{k \in \mathbf{D}}\langle p(x), k\rangle .
\end{gathered}
$$

Recalling that $\sup _{k \in c o(\mathbf{D})}\langle p(x), k\rangle=\sup _{k \in \mathbf{D}}\langle p(x), k\rangle$, we obtain a contradiction.

b) To prove the theorem, suppose, by contradiction, that there exists a nontrivial $\eta \in W_{0}^{1, \infty}(\Omega)$, that verifies condition (1) almost everywhere.

In the case that $\operatorname{int}(\operatorname{co}(\mathbf{D}))=\varnothing, \mathbf{D}$ is contained in a hyperplane, and condition (1) implies that also $\nabla \eta$ is in a hyperplane, a contradiction to Lemma 1 . Hence, in what follows, we consider $\operatorname{int}(\operatorname{co}(\mathbf{D})) \neq \varnothing$.

c) Claim. For every $x \in \Omega$, there exists $c$ such that $\eta(S(t ; x))=c$ for $t \in\left(\alpha_{x}, \beta_{x}\right)$, the maximal interval of existence for the solution $S$.

Proof of this claim. By assumption, for almost every $x \in \Omega$,

$$
\langle\nabla u(x), p(x)\rangle=\sup _{k \in \mathbf{D}}\langle k, p(x)\rangle
$$


and

$$
\begin{aligned}
\langle\nabla \eta(x), p(x)\rangle & =\langle-\nabla u(x), p(x)\rangle+\langle\nabla \eta(x)+\nabla u(x), p(x)\rangle \leq \\
& -\langle\nabla u(x), p(x)\rangle+\sup _{k \in \mathbf{D}}\langle k, p(x)\rangle,
\end{aligned}
$$

so that

$$
\langle\nabla \eta(x), p(x)\rangle \leq 0 .
$$

Since $\eta \in W_{0}^{1, \infty}(\Omega)$, the assumption on the divergence of $p$ implies

$$
\int_{\Omega}\langle\nabla \eta(x), p(x)\rangle \mathrm{d} x=0,
$$

hence we obtain that, for almost every $x \in \Omega$,

$$
\langle\nabla \eta(x), p(x)\rangle=0 .
$$

Fix $x^{*} \in \Omega$. Consider the $N-1$ dimensional affine space

$$
V=x^{*}+p\left(x^{*}\right)^{\perp} .
$$

There exists $\delta>0$ and $r>0$, such that a solution $S(t ; v)$ to $\dot{x}=p(x)$ and $x(0)=v$ exists for $v \in V \cap B\left(x^{*}, r\right)$ on an interval $(-\delta, \delta)$. The map $(t ; v) \rightarrow S(t ; v)$ is Lipschitzian and invertible. Hence, by the coarea theorem, with the exception of a subset of $V$ of $N-1$ dimensional measure zero, $S(t ; v)$ meets the set $M$, where $\langle\nabla \eta(x), p(x)\rangle \neq 0$, on a subset of $(-\delta, \delta)$ of 1-dimensional measure zero, and, outside of this exceptional set, we have

$$
\frac{\mathrm{d}}{\mathrm{d} t} \eta(S(t ; v))=\langle\nabla \eta(S(t ; v)), p(S(t ; v))\rangle=0 .
$$

Hence, there exists a sequence $v_{n} \rightarrow x^{*}$ such that $\eta\left(S\left(t ; v_{n}\right)\right) \equiv c_{n}$ on $(-\delta, \delta)$. Since the limit of solutions is a solution and $\eta$ is continuous, we have that $\eta\left(S\left(t ; x^{*}\right)\right) \equiv c$ on $(-\delta, \delta)$. This local reasoning can be extended to the maximal interval of existence, proving the claim.

d) Let $\bar{x}$ be such that $\eta(\bar{x})>0$, and define

$$
E=\left\{x \in \Omega: \eta(x) \geq \frac{1}{2} \eta(\bar{x})\right\} \subset \Omega .
$$

The set $E$ is nonempty, compact, $\operatorname{int}(E) \neq \varnothing$ and $d(E, \partial \Omega)>0$. As a consequence of c), it cannot happen that there exists $x \in E$ such that, for some $t \in\left(\alpha_{x}, \beta_{x}\right), S(t ; x) \notin E$. Hence for every $x \in E$ and every $t \in\left(\alpha_{x}, \beta_{x}\right)$, $S(t ; x) \in E$. By the basic theorems on the prolongability of solutions to ordinary differential equations, it follows then that the solution $S(t ; x)$ must be defined for every $t \in \mathbb{R}$, since $d(E, \partial \Omega)>0$. Hence, for every $t \in \mathbb{R}$, the map $S(t ; \cdot)$ is a bijection of $E$ into itself and, in addition, by Lemma 1, it is measure preserving.

e) We wish to apply the following Poincaré recurrence Theorem to the map $S(t ; \cdot)$, (see for instance [1] for the proof).

Lemma 3 (Poincaré). Let $E$ be a compact, nonempty set such that $\operatorname{int}(E) \neq \varnothing$, and let $\psi: E \rightarrow E$ a bijective, measure preserving function. Then, for every $x_{0} \in \operatorname{int}(E)$ and every $\varepsilon>0$, there exists an integer $k>0$ such that

$$
\psi^{k}\left(B\left(x_{0}, \varepsilon\right)\right) \cap B\left(x_{0}, \varepsilon\right) \neq \varnothing .
$$


Going back to the proof, let $r^{0}>0$ and $x^{0}$ be such that $B\left(x^{0}, r^{0}\right) \subset E$ and let $t_{0}>0$ be such that $S\left(t_{0} ; x^{0}\right) \neq x^{0}$. Let $V \subset \Omega$ be a neighborhood of the trajectory

$$
\left\{S\left(t ; x^{0}\right): t \in\left[0, t_{0}\right]\right\}
$$

and let $p^{0}>0$ be such that $\|p(x)\| \geq p^{0}$ for $x$ in $V$. Let $r \leq r^{0}$ be so small that:

$$
S\left(t_{0} ; B\left(x^{0}, r\right)\right) \cap B\left(x^{0}, r\right)=\varnothing
$$

and, for every $\xi \in B\left(x^{0}, r\right)$, the solution $S(t ; \xi) \in V$ for $t \in\left[0, t_{0}\right]$. Applying Poincaré's method we obtain that, for every $\rho<r$, there exist $\xi_{\rho} \in B\left(x^{0}, \rho\right)$ and an integer $\nu_{\rho}>1$, such that

$$
\left|S\left(t_{0} \nu_{\rho} ; \xi_{\rho}\right)-x^{0}\right| \leq \rho \text {. }
$$

f) Choose $v \in \operatorname{int}(\mathbf{D})$ and let $s>0$ be such that $B(v, s) \subset \mathbf{D}$. Consider the function $u_{0}$ defined by

$$
u_{0}(x)=u(x)-\langle v, x\rangle .
$$

Condition (2) implies that $u_{0}$, computed along $S(t ; x)$, for $x \in B\left(x^{0}, r\right)$, is strictly increasing:

$$
\begin{gathered}
\frac{\mathrm{d}}{\mathrm{d} t} u_{0}(S(t ; x))=\langle\nabla u(S(t ; x))-v, p(S(t ; x))\rangle=\sup _{k \in D}\langle k-v, p(S(t ; x))\rangle \\
\geq s\|p(S(t ; x))\|>0 ;
\end{gathered}
$$

in particular, for $\xi \in B\left(x^{0}, \rho\right)$, with $\rho \leq r$, we obtain

$$
u_{0}\left(S\left(t_{0} \nu_{\rho} ; \xi\right)\right)-u_{0}(\xi) \geq t_{0} \nu_{\rho} s p^{0} \geq t_{0} s p^{0} .
$$

This last estimate is independent of $\rho$.

Apply this estimate to $\xi_{\rho}$; we have that both $\xi_{\rho}$ and $S\left(t_{0} \nu_{\rho} ; \xi_{\rho}\right)$ are in $B\left(x^{0}, \rho\right)$. By the continuity of $u_{0}$ at $x^{0}$, the difference $u_{0}\left(S\left(t_{0} \nu_{\rho} ; \xi_{\rho}\right)\right)-u_{0}\left(\xi_{\rho}\right)$ can be made arbitrarily small by decreasing $\rho$, a contradiction.

The following result completes the discussion of the example in Section 2.

Theorem 3. Let $\Omega \subset \mathbb{R}^{2}$ be the open disk $x_{1}^{2}+x_{2}^{2}<1$ and $v\left(x_{1} ; x_{2}\right)=\sqrt{x_{1}^{2}+x_{2}^{2}}$. There is no vector function $p \in L_{\text {loc }}^{1}(\Omega), p(x) \neq 0$ a.e., such that $\operatorname{div}(p)=0$, and

$$
\langle p(x), \nabla v(x)\rangle=\sup _{k \in \mathbf{B}}\langle p(x), k\rangle
$$

for almost every $x \in \Omega$.

Proof of Theorem 3. The function $\eta=-\sqrt{x_{1}^{2}+x_{2}^{2}}+1$ is in $W_{0}^{1, \infty}(\Omega)$ and is a solution to the differential inclusion

$$
\nabla \eta(x) \in-\nabla v(x)+\mathbf{B} .
$$

Assume that $p$ exists. By assumption we must have, for almost every $x \in \Omega$,

$$
\langle p(x), \nabla v(x)\rangle=\|p(x)\|
$$

so that $p(x)=\alpha(x) \frac{x}{\|x\|}$, and $\alpha \geq 0$. On the other hand, in c) of the proof of the previous theorem we have obtained that, for almost every $x \in \Omega$,

so that $\alpha(x)=0$ a.e. in $\Omega$.

$$
\langle\nabla \eta(x), p(x)\rangle=0,
$$




\section{When $\mathbf{D}=\mathbf{B}$, not a) implies b)}

We prove this part of the conjecture in the case $\mathbf{D}=\mathbf{B}$.

Theorem 4. Let $u \in W^{1, \infty}(\Omega)$ be a solution to $\nabla u(x) \in \mathbf{B}$ and assume that there exist no nontrivial $\eta \in$ $W_{0}^{1, \infty}(\Omega)$, solution to the differential inclusion

$$
\nabla \eta(x) \in-\nabla u(x)+\mathbf{B}
$$

Then:

i) the solution $u$ belongs to $C^{1}(\Omega) \cap W_{\text {loc }}^{2, \infty}(\Omega)$;

ii) there exists $p \in L_{\text {loc }}^{1}(\Omega), p(x) \neq 0$ for almost every $x \in \Omega$, such that $\operatorname{div}(p)=0$, and

$$
\langle p(x), \nabla u(x)\rangle=\sup _{k \in B}\langle p(x), k\rangle
$$

for almost every $x \in \Omega$.

Remark 1. In the proof of Theorem 4, we will construct a function $p$ that verifies ii). This function $p$ can be interpreted as a mass-transfer vector field, and from condition (2) we see that $\nabla u$ determines the optimal direction for $p$. Hence, we expect $p$ to be of the form $p=\lambda \nabla u$, for a suitable function $\lambda(x)$, and we compute $\lambda$ by the equation $\operatorname{div}(\lambda \nabla u)=0$. As appears in [7], this equation is related to the Monge-Kantorovich transport problem. In particular, $\lambda$ plays the role of a transport density, and is the Lagrange multiplier for the constraint $\nabla u \in \mathbf{B}$.

Proof of Theorem 4. The proof makes use of some results and techniques developed in [5].

a) Fix any point $x^{0} \in \Omega$. Using Lemmas 2.2 and 2.3 of [5], from the fact that there is no variation $\eta$ such that $u\left(x^{0}\right)+\eta\left(x^{0}\right)<u\left(x^{0}\right)$, we infer the existence of at least one unit vector, a direction, $d^{+}$, with the property that, for every $r$ such that the ball $B\left(x^{0}, r\right)$ is contained in $\Omega$, we have $u\left(x^{0}+r d^{+}\right)-u\left(x^{0}\right)=r$. Such a direction will be called a direction of maximal growth. By the same reasons, since there is no variation $\eta$ such that $u\left(x^{0}\right)+\eta\left(x^{0}\right)>u\left(x^{0}\right)$, we infer the existence of at least one direction, $d^{-}$, such that $u\left(x^{0}\right)-u\left(x^{0}+r d^{-}\right)=r$. However we must have that $d^{+}=d^{-}$, in fact, since $u$ is Lipschitzian of constant 1 , we have

$$
r\left\|d^{+}+d^{-}\right\| \geq\left|u\left(x^{0}+r d^{+}\right)-u\left(x^{0}-r d^{-}\right)\right|=\left|u\left(x^{0}+r d^{+}\right)-u\left(x^{0}\right)-u\left(x^{0}+r d^{-}\right)+u\left(x^{0}\right)\right|=2 r,
$$

i.e. $\left\|d^{+}+d^{-}\right\|=\left\|d^{+}\right\|+\left\|d^{-}\right\|$, that implies $d^{+}=d^{-}$. Notice that this result implies that $d^{+}$and $d^{-}$ are unique. Hence, from the assumption that there is no variation $\eta$, to each $x \in \Omega$ we associate a unique direction $d(x)$ such that $u(x+r d(x))-u(x)=r$ as long as $x+r d(x) \in \Omega$; i.e., there exists a unique segment $(x+\alpha(x) d(x), x+\beta(x) d(x)), \alpha(x)<0<\beta(x)$, such that: $x+\alpha(x) d(x) \in \partial \Omega, x+\beta(x) d(x) \in \partial \Omega$ and $u\left(x+\lambda_{1} d(x)\right)-u\left(x+\lambda_{2} d(x)\right)=\lambda_{1}-\lambda_{2}$ for every $\lambda_{1}, \lambda_{2} \in(\alpha(x), \beta(x))$. The direction $d$ has the following interpretation: at every point $x^{0}$ such that $\nabla u\left(x^{0}\right)$ exists, we have that $\nabla u\left(x^{0}\right)=d\left(x^{0}\right)$. In fact, from

$$
u(x)-u\left(x^{0}\right)=\left\langle\nabla u\left(x^{0}\right), x-x^{0}\right\rangle+\left\|x-x^{0}\right\| o\left(\left\|x-x^{0}\right\|\right),
$$

we obtain

$$
r=r\left\langle\nabla u\left(x^{0}\right), d\left(x^{0}\right)\right\rangle+r o(r),
$$

that implies $\nabla u\left(x^{0}\right)=d\left(x^{0}\right)$. Moreover, the following property holds: for no $y \in \Omega$ we can have

$$
y=x+\lambda \mathrm{d}(x)=x^{\prime}+\lambda^{\prime} d\left(x^{\prime}\right)
$$

unless $d\left(x^{\prime}\right)=d(x)$. In fact, otherwise, both $d(x)$ and $d\left(x^{\prime}\right)$ would be directions of maximal growth at $y$, contradicting the uniqueness of $d(y)$. 
b) Claim. Let $\rho$ be such that $B\left(x^{0}, \rho\right) \subset \Omega$. Then, on $B\left(x^{0}, \frac{\rho}{6}\right)$, the map $x \rightarrow \mathrm{d}(x)$ is Lipschitzian of constant $\Lambda=\frac{3}{\rho}$.

Proof of this Claim. Let $P$ and $P^{\prime}$ in $B\left(x^{0}, \frac{\rho}{6}\right)$, so that $\left\|P-P^{\prime}\right\| \leq \frac{\rho}{3}$. Set $d=d(P)$ and $d^{\prime}=d\left(P^{\prime}\right)$; let $O$ on the line $r=\{P+\lambda d\}$ and $O^{\prime}$ on the line $r^{\prime}=\left\{P^{\prime}+\lambda d^{\prime}\right\}$ be such that $\left\|O-O^{\prime}\right\|=\inf _{Q \in r, Q^{\prime} \in r^{\prime}}\left\|Q-Q^{\prime}\right\|$. We have that $\left(O-O^{\prime}\right)$ is orthogonal both to $r$ and to $r^{\prime}$. Two cases are possible: either, a), inf $\left\{\|P-O\|,\left\|P^{\prime}-O^{\prime}\right\|\right\}>\frac{\rho}{3}$ or, b), inf $\left\{\|P-O\|,\left\|P^{\prime}-O^{\prime}\right\|\right\} \leq \frac{\rho}{3}$.

Consider case a). Call $P$ the point such that $\|P-O\| \leq\left\|P^{\prime}-O^{\prime}\right\|$. We will need the line $r^{\prime \prime}=r^{\prime}+\left(O-O^{\prime}\right)$ : it is the parallel to $r^{\prime}$ in the plane containing $r$ and orthogonal to $\left(O-O^{\prime}\right)$. Let $P^{\prime \prime}$ be the projection of $P^{\prime}$ on $r^{\prime \prime}$. Since $\left\|P^{\prime \prime}-O\right\|=\left\|P^{\prime}-O^{\prime}\right\| \geq\|P-O\|$, on the segment $\left[O, P^{\prime \prime}\right]$ choose $P_{i}$ such that $\left\|P_{i}-O\right\|=\|P-O\|$ and consider the isosceles triangle $O, P^{\prime}, P_{i}$ : we have

$$
\frac{\left\|d-d^{\prime}\right\|}{1}=\frac{\left\|P_{i}-P\right\|}{\|P-O\|}
$$

so that $\|P-O\| \geq \frac{\rho}{3}$ implies

$$
\left\|d-d^{\prime}\right\| \leq \frac{3}{\rho}\left\|P_{i}-P\right\| .
$$

We claim that $\left\|P^{\prime \prime}-P\right\| \geq\left\|P_{i}-P\right\|$. In fact, the angle $P, P_{i}, P^{\prime \prime}$ is larger than $\frac{\pi}{2}$, being the triangle $O, P^{\prime}, P_{i}$ isosceles, so that

$$
\begin{aligned}
\left\|P^{\prime \prime}-P\right\|^{2} & =\left\|P-P_{i}\right\|^{2}+\left\|P_{i}-P^{\prime \prime}\right\|^{2}+2\left\langle P-P_{i}, P_{i}-P^{\prime \prime}\right\rangle \\
& \geq\left\|P-P_{i}\right\|^{2}+\left\|P_{i}-\left.P^{\prime \prime}\right|^{2} \geq\right\| P-P_{i} \|^{2} .
\end{aligned}
$$

We have shown that

$$
\left\|d-d^{\prime}\right\| \leq \frac{3}{\rho}\left\|P_{i}-P\right\| \leq \frac{3}{\rho}\left\|P^{\prime \prime}-P\right\| \leq \frac{3}{\rho}\left\|P^{\prime}-P\right\| .
$$

Consider case b). Consider the two points $O$ and $O^{\prime}$; since $\left\|O-O^{\prime}\right\| \leq\left\|P-P^{\prime}\right\|$, we obtain that both points $O$ and $O^{\prime}$ are in $B\left(x^{0}, \rho\right)$, so that $u$ is defined at $O$ and $O^{\prime}$. For case b), we assign names to the points $P$ and $P^{\prime}$ by assuming that $u\left(O^{\prime}\right) \geq u(O)$. With this choice of names, consider again the lines $r, r^{\prime}$ and set again $r^{\prime \prime}=r^{\prime}+\left(O-O^{\prime}\right)$. On $r$ consider the segment $[A, D]$, centered at $O$, such that $\|A-O\|=\|D-O\|=\frac{\rho}{3}$; on $r^{\prime}$, the segment $\left[B^{\prime}, C^{\prime}\right]$, centered at $O^{\prime}$, such that $\left\|B^{\prime}-O^{\prime}\right\|=\left\|C^{\prime}-O^{\prime}\right\|=\frac{\rho}{3}$; orientations are chosen so that $A=O+\frac{\rho}{3} d$ and $B^{\prime}=O^{\prime}+\frac{\rho}{3} d^{\prime}$. Call $B$ and $C$ the projections of $B^{\prime}$ and $C^{\prime}$ on the line $r^{\prime \prime}$. We obtain

$$
\begin{aligned}
\left\|B^{\prime}-D\right\| & \geq u\left(B^{\prime}\right)-u(D)=u\left(B^{\prime}\right)-u\left(O^{\prime}\right)+u\left(O^{\prime}\right)-u(O)+u(O)-u(D) \\
& \geq u\left(B^{\prime}\right)-u\left(O^{\prime}\right)+u(O)-u(D)=\frac{\rho}{3}+\frac{\rho}{3} .
\end{aligned}
$$

Set $H=\frac{1}{2} A+\frac{1}{2} B$. We have:

$$
\begin{aligned}
\|H-O\|^{2} & =\left\|\frac{1}{2}(A-O)+\frac{1}{2}(B-O)\right\|^{2}=\left\|\frac{1}{2}(O-D)+\frac{1}{2}(B-O)\right\|^{2} \\
& =\left\|\frac{1}{2}(B-D)\right\|^{2}=\frac{1}{4}\left(\left\|B^{\prime}-D\right\|^{2}-\left\|O-O^{\prime}\right\|^{2}\right)
\end{aligned}
$$


the last equality deriving from the Pytagorean Theorem applied to the triangle $D, B, B^{\prime}$. Hence we have:

$$
\begin{aligned}
\frac{1}{4}\|A-B\|^{2} & =\|B-H\|^{2}=\|B-O\|^{2}-\|H-O\|^{2} \\
& =\left(\frac{\rho}{3}\right)^{2}-\frac{1}{4}\left(\left\|B^{\prime}-D\right\|^{2}-\left\|O-O^{\prime}\right\|^{2}\right) \leq\left(\frac{\rho}{3}\right)^{2}-\frac{1}{4}\left(\frac{2 \rho}{3}\right)^{2}+\frac{1}{4}\left\|O-O^{\prime}\right\|^{2} \\
& =\frac{1}{4}\left\|O-O^{\prime}\right\|^{2} .
\end{aligned}
$$

We obtain

$$
\|A-B\|=\left\|O+\frac{\rho}{3} d-\left(O+\frac{\rho}{3} d^{\prime}\right)\right\| \leq\left\|O-O^{\prime}\right\| \leq\left\|P-P^{\prime}\right\| .
$$

We conclude that, for case b) as well, we have

$$
\left\|d-d^{\prime}\right\| \leq \frac{3}{\rho}\left\|P-P^{\prime}\right\|
$$

proving the claim.

c) We claim that, as a consequence of the Lipschitzianity of $d$, we have that $u \in C^{1}(\Omega) \cap W_{l o c}^{2, \infty}(\Omega)$. The directions of the coordinate axis are denoted by $e_{i}$.

Fix $x$; let $B(x, r) \subset \Omega$ and let $\Lambda$ be a Lipschitz constant for $d$ in $B(x, r)$. We first notice that if it happens that on the intersection of the line $\left\{x+t e_{i}: t \in \mathbb{R}\right\}$ with $B(x, r), u$ is differentiable at $x+t e_{i}$ for almost every $t$, then we must have

$$
\left|u\left(x+h e_{i}\right)-u(x)-h\left\langle d(x), e_{i}\right\rangle\right| \leq h^{2} \Lambda .
$$

In fact, the Lipschitzian map $t \rightarrow u\left(x+t e_{i}\right)$ is the integral of its derivative, that coincides, for a.e. $t$, with $\left\langle d\left(x+t e_{i}\right), e_{i}\right\rangle$, so that

$$
\left|u\left(x+h e_{i}\right)-u(x)-h\left\langle d(x), e_{i}\right\rangle\right|=\left|h \int_{0}^{1}\left\langle d\left(x+s h e_{i}\right)-d(x), e_{i}\right\rangle \mathrm{d} s\right| \leq h^{2} \Lambda .
$$

Notice next that, since $\nabla u(x)$ exists for a.e. $x \in \Omega$, there must exists a sequence $x_{n} \rightarrow x$ such that, on the intersection of the line $\left\{x_{n}+t e_{i}: t \in \mathbb{R}\right\}$ with $B(x, r), \nabla u\left(x_{n}+t e_{i}\right)$ exists for a.e. $t$. Then we have:

$$
\begin{aligned}
\left|u\left(x+h e_{i}\right)-u(x)-h\left\langle d(x), e_{i}\right\rangle\right|= & \mid u\left(x_{n}+h e_{i}\right)-u\left(x_{n}\right)-h\left\langle d\left(x_{n}\right), e_{i}\right\rangle+h\left\langle d\left(x_{n}\right)-d(x), e_{i}\right\rangle \\
& +u\left(x+h e_{i}\right)-u\left(x_{n}+h e_{i}\right)+u\left(x_{n}\right)-u(x) \mid \\
& \leq h^{2} \Lambda+h \Lambda\left|x_{n}-x\right|+2\left|x_{n}-x\right| .
\end{aligned}
$$

Letting $n \rightarrow \infty$ we obtain that $\frac{\partial u}{\partial x_{i}}$ exists at $x$ and equals $\left\langle d(x), e_{i}\right\rangle$. Since the gradient is continuous, we obtain that $u$ is differentiable and that $u \in C^{1}(\Omega)$.

Fix $\eta \in C_{c}^{\infty}(\Omega)$. Then on $\operatorname{supp}(\eta), \nabla u(x)=d(x)$ is uniformly Lipschitzian: hence, see [8], for each component $d^{i}$ and each $j$ there is $g_{j}^{i}$ such that

This proves i).

$$
\int_{\Omega} g_{j}^{i} \eta \mathrm{d} x=-\int_{\Omega} d^{i} \frac{\partial \eta}{\partial x_{j}} \mathrm{~d} x .
$$

d) As established in the Remark, the map $p$, as required in ii), will be of the form $\lambda(x) d(x)$. To find $\lambda$ amounts to finding a weak solution to the equation $\operatorname{div}(\lambda(x) d(x))=0$, where $\operatorname{div}(d(x)) \in L_{\text {loc }}^{\infty}(\Omega)$.

Fix $x^{*} \in \Omega$ and consider the corresponding level set for the function $u$, i.e. $\left\{x: u(x)=u\left(x^{*}\right)\right\}$. We claim that we can parametrize locally this set by a differentiable and invertible map $\phi_{x^{*}}$ from an open set $V_{x^{*}}$ in 
a $N-1$ space, to $\Omega$, i.e. that there exists $V_{x^{*}}, \phi_{x^{*}}, r^{*}$ such that $u\left(\phi_{x^{*}}(\xi)\right) \equiv u\left(x^{*}\right)$, for every $\xi \in V_{x^{*}}$ and $\phi\left(V_{x^{*}}\right)=\left\{u(x)=u\left(x^{*}\right)\right\} \cap B\left(x^{*}, r^{*}\right)$.

Proof of this Claim. Consider the $N-1$ dimensional space $d\left(x^{*}\right)^{\perp}$, defined by the equation $\left\langle d\left(x^{*}\right), x\right\rangle=$ $\left\langle d\left(x^{*}\right), x^{*}\right\rangle$; let $d_{i}\left(x^{*}\right) \neq 0$, set the $N-1$ vector $\xi$ be $\xi_{j}=x_{j}, j \neq i$, and set $\xi^{*}$ be $\xi_{j}^{*}=x_{j}^{*}, j \neq i$, so that $d\left(x^{*}\right)^{\perp}$ is the image of the affine map $\ell$, given by $\ell(\xi)_{j}=x_{j}, j \neq i$, and

$$
\ell(\xi)_{i}=\frac{\left\langle d\left(x^{*}\right), x^{*}\right\rangle-\sum_{j \neq i} d_{j}\left(x^{*}\right) \xi_{j}}{d_{i}\left(x^{*}\right)}
$$

The map $\ell$ is one to one from $\mathbb{R}^{N-1}$ to $\mathbb{R}^{N}$. For $\xi$ in a sufficiently small neighborhood $V_{x^{*}}$ of $\xi^{*}$, so that the maps are defined, we have that $u(\ell(\xi)+t d(\ell(\xi)))=u(\ell(\xi))+t$ and $u(\ell(\xi)+t d(\ell(\xi)))$ assumes the value $u\left(x^{*}\right)$ for $u\left(x^{*}\right)-u(\ell(\xi))$. The required parametrization is given by the (differentiable) map $\phi_{x^{*}}(\xi)=\ell(\xi)+\left(u\left(x^{*}\right)-\right.$ $u(\ell(\xi))) d(\ell(\xi))$. The map $\phi_{x^{*}}$ is invertible: assume that $\ell(\xi)+t(\xi) d(\ell(\xi))=\ell\left(\xi^{\prime}\right)+t\left(\xi^{\prime}\right) d\left(\ell\left(\xi^{\prime}\right)\right)=P$; then $u(P)-u\left(x^{*}\right)=t(\xi), u(P)-u\left(x^{*}\right)=t\left(\xi^{\prime}\right)$ and, by the results of a), $d(\ell(\xi))=d\left(\ell\left(\xi^{\prime}\right)\right)$, so that $\ell(\xi)=\ell\left(\xi^{\prime}\right)$ and $\xi=\xi^{\prime}$.

e) Consider the flow $S(t ; x)=x+t d(x)$ : it is a solution to the Cauchy problem

$$
\frac{d}{\mathrm{~d} t} S(t ; x)=d(S(t ; x)), \quad S(0 ; x)=x .
$$

In particular, consider the map $(t ; \xi) \rightarrow S\left(t ; \phi_{x^{*}}(\xi)\right)$ : by the basic theorems on uniqueness for ordinary differential equations, and by the invertibility of $\phi_{x^{*}}$, it is an invertible map.

We will denote by $D$ the square matrix of partial derivatives of the vector field $d(x)$ and by $M_{x}(t)$ the square matrix of partial derivatives of $S(t ; x)$ with respect to the space variables, computed at $x$, i.e. $M_{x}(t)=I+t D(x)$. Since the vector field $d$ is autonomous, we have the basic identity

$$
M_{x}(t) d(x)=d(S(t ; x)) .
$$

In addition, Lindelöf's Theorem on differentiability with respect to initial conditions implies that

$$
\operatorname{det}\left(M_{x}(t)\right)=\mathrm{e}^{\int_{0}^{t} \operatorname{tr} D(s) \mathrm{d} s}
$$

where the trace of $D$ appearing at the right hand side is computed along $S(s ; x)$. As a consequence of the uniform Lipschitzianity of $d$ on compact subsets of $\Omega$, we have that on a compact set, there exists $k$ such that $\operatorname{det}\left(M_{x}(t)\right) \geq k>0$. Denote by $\Phi_{\xi}$ the $N \times(N-1)$ matrix of partial derivatives of $\phi$ with respect to $\xi$. We obtain that

$$
D_{(t ; \xi)}(S(t ; \phi(\xi)))=\left(d(S(t ; \phi(\xi))) ; M_{\phi(\xi)}(t) \Phi_{\xi}\right)
$$

and, recalling that $\mathrm{d}(S(t ; \phi(\xi)))=d(\phi(\xi))=M_{\phi(\xi)}(t) d(\phi(\xi))$, we obtain

$$
\operatorname{det}\left(D_{(t ; \xi)}(S(t ; \phi(\xi)))\right)=\operatorname{det}\left(M_{\phi(\xi)}(t)\right) \operatorname{det}\left(d(\phi(\xi)) ; \phi_{\xi_{1}} ; \ldots ; \phi_{\xi_{N-1}}\right) .
$$

f) An easy contradiction argument shows that the set

$$
O_{x^{*}}=\left\{(t ; \xi): \alpha\left(\phi_{x^{*}}(\xi)\right)<t<\beta\left(\phi_{x^{*}}(\xi)\right) ; \xi \in V_{x^{*}}\right\}
$$

is an open subset of $\mathbb{R} \times \mathbb{R}^{N-1}$ and, being the continuous map $S\left(t ; \phi_{x^{*}}(\xi)\right)$ one to one, its image $\mathrm{S}_{x^{*}}$ is an open subset of $\Omega$. 
Consider a countable covering of $\Omega$ by sets $\mathrm{S}_{x_{n}}, n=1, \ldots$ (for brevity we will set $\mathrm{S}_{x_{n}}=\mathrm{S}_{n}, V_{x_{n}}=V_{n}$ and $\left.\phi_{x_{n}}=\phi_{n}\right)$. Fix $x \in \mathrm{S}_{n}$; let $t$ and $\xi$ be such that $x=S\left(t ; \phi_{n}(\xi)\right)$ and set

$$
\lambda_{n}(x)=\frac{1}{\operatorname{det} M_{\phi_{n}(\xi)}(t)} .
$$

This definition sets (arbitrarily) $\lambda_{n}$ to be 1 on the level set $\left\{x: u(x)=u\left(x_{n}\right)\right\} \cap \mathrm{S}_{n}$. Set $E_{1}=\Omega \cap \mathrm{S}_{1} ; E_{n+1}=$ $\Omega \cap\left[\mathrm{S}_{n+1} \backslash E_{n}\right]$, so that $\Omega=\bigcup E_{n}$, and the $E_{n}$ are disjoint.

In general, define $\lambda(x)=\sum \lambda_{n}(x) \chi_{E_{n}}$. On a compact subset of $\Omega$, we have that $\lambda_{n}(x) \leq h$ where $h$ does not depend on $n$, so that $\lambda \in L_{\text {loc }}^{\infty}(\Omega)$. We claim that, for every $\eta \in C_{c}^{\infty}(\Omega)$, we have

$$
\int_{\Omega} \lambda(x)\langle d(x), \nabla \eta(x)\rangle \mathrm{d} x=\sum_{n} \int_{E_{n}} \lambda_{n}(x)\langle d(x), \nabla \eta(x)\rangle \mathrm{d} x=0
$$

i.e. that the map $p(x)=\lambda(x) d(x)$ has divergence zero.

On $E_{n}$ consider the change of variables given by $x=S\left(t ; \phi_{n}(\xi)\right)$, with Jacobian $J_{n}(t ; \xi)=\left|\operatorname{det} D_{(t ; \xi)}(S(t ; \phi(\xi)))\right|$. We have

$$
\begin{aligned}
\lambda_{n}\left(S\left(t ; \phi_{n}(\xi)\right)\right) J_{n}(t ; \xi) & =\frac{1}{\operatorname{det} M_{\phi_{n}(\xi)}(t)}\left|\operatorname{det} M_{\phi(\xi)}(t) \operatorname{det}\left(d(\phi(\xi)) ; \phi_{\xi_{1}} ; \ldots ; \phi_{\xi_{n-1}}\right)\right| \\
& =\left|\operatorname{det}\left(d(\phi(\xi)) ; \phi_{\xi_{1}} ; \ldots ; \phi_{\xi_{n-1}}\right)\right|
\end{aligned}
$$

so that

$$
\begin{aligned}
& \int_{E_{n}} \lambda_{n}(x)\langle d(x),\nabla \eta(x)\rangle \mathrm{d} x=\int_{E_{n}} \lambda_{n}\left(S\left(t ; \phi_{n}(\xi)\right)\right)\left\langle d\left(S\left(t ; \phi_{n}(\xi)\right)\right), \nabla \eta\left(S\left(t ; \phi_{n}(\xi)\right)\right)\right\rangle J_{n}(t ; \xi) d(t ; \xi) \\
&=\int\left(\int_{\alpha\left(\phi_{n}(\xi)\right)}^{\beta\left(\phi_{n}(\xi)\right)} \lambda_{n}\left(S\left(t ; \phi_{n}(\xi)\right)\right)\left\langle d\left(S\left(t ; \phi_{n}(\xi)\right)\right), \nabla \eta\left(S\left(t ; \phi_{n}(\xi)\right)\right)\right\rangle J_{n}(t ; \xi) \mathrm{d} t\right) \mathrm{d} \xi \\
&=\int\left(\int_{\alpha\left(\phi_{n}(\xi)\right)}^{\beta\left(\phi_{n}(\xi)\right)} \frac{\mathrm{d}}{\mathrm{d} t} \eta\left(S\left(t ; \phi_{n}(\xi)\right)\right) \mathrm{d} t\right)\left|\operatorname{det}\left(d(\phi(\xi)) ; \phi_{\xi_{1}} ; \ldots ; \phi_{\xi_{n-1}}\right)\right| \mathrm{d} \xi
\end{aligned}
$$

Since, for every $\xi, S\left(\alpha\left(\phi_{n}(\xi)\right) ; \phi_{n}(\xi)\right)$ and $S\left(\beta\left(\phi_{n}(\xi)\right) ; \phi(\xi)\right)$ belong to $\partial \Omega$, we obtain that $\eta(S(\alpha(\phi(\xi)) ; \phi(\xi)))=$ $\eta(S(\beta(\phi(\xi)) ; \phi(\xi)))=0$ for every $\xi$, so that

$$
\int_{\Omega} \lambda(x)\langle d(x), \nabla \eta(x)\rangle \mathrm{d} x=0 .
$$

g) We have

concluding the proof.

$$
\langle p(x), \nabla u(x)\rangle=\langle\lambda(x) d(x), \nabla u(x)\rangle=\lambda(x)=\sup _{k \in B}\langle p(x), k\rangle
$$

Remark 2. The vector $p(\cdot)$ admits a divergence in the integral sense, but need not belong to $W_{l o c}^{1,1}(\Omega)$.

In fact, in $\mathbb{R}^{2}$ consider

$$
\Omega=\left\{(x ; y): x^{2}+y^{2}<1, x \leq 0, y>0\right\} \cup\left\{(x ; y): x^{2}+(y-1)^{2}<1, x \geq 0, y<1\right\} .
$$

On $\Omega$ set $P=(x ; y)$ and

$$
u(P)=\left\{\begin{array}{cc}
\sqrt{x^{2}+y^{2}} & \text { if } \quad x \leq 0 \\
1-\sqrt{x^{2}+(y-1)^{2}} & \text { otherwise }
\end{array}\right.
$$


Then

and

$$
\nabla u(P)=\left\{\begin{array}{cl}
\frac{P}{\|P\|} & \text { if } x \leq 0 \\
\frac{(0 ; 1)-P}{\|P-(0 ; 1)\|} & \text { otherwise }
\end{array}\right.
$$

One verifies that the differential equation for $\lambda$

$$
\Delta u(P)=\left\{\begin{array}{cc}
\frac{1}{\|P\|} & \text { if } \quad x \leq 0 \\
\frac{-1}{\|P-(0 ; 1)\|} & \text { otherwise. }
\end{array}\right.
$$

$$
\langle\nabla \lambda(P), \nabla u(P)\rangle+\lambda(P) \Delta u(P)=0
$$

admits the solution

Hence

$$
\lambda(P)=\left\{\begin{array}{cl}
\frac{1}{\|P\|} & \text { if } x \leq 0 \\
\frac{1}{\|P-(0 ; 1)\|} & \text { otherwise. }
\end{array}\right.
$$

$$
p(P)=\left\{\begin{array}{cc}
\frac{P}{\|P\|^{2}} & \text { if } x \leq 0 \\
\frac{(0 ; 1)-P}{\|P-(0 ; 1)\|^{2}} & \text { otherwise }
\end{array}\right.
$$

that has a jump discontinuity through the line $x=0$. Hence $p$ cannot belong to $W^{1,1}(\Omega)$.

\section{REFERENCES}

[1] V.I. Arnold, Mathematical methods of classical mechanics, Graduate Texts in Mathematics 60, Springer-Verlag, New York, Heidelber, Berlin.

[2] H. Brezis, Analyse fonctionnelle, théorie et applications. Masson, Paris (1983).

[3] A. Cellina, On minima of a functional of the gradient: necessary conditions. Nonlinear Anal. 20 (1993) 337-341.

[4] A. Cellina, On minima of a functional of the gradient: sufficient conditions. Nonlinear Anal. 20 (1993) 343-347.

[5] A. Cellina and S. Perrotta, On the validity of the maximum principle and of the Euler-Lagrange equation for a minimum problem depending on the gradient. SIAM J. Control Optim. 36 (1998) 1987-1998.

[6] L.C. Evans, Partial Differential Equations, Graduate Studies in Mathematics 19, American Mathematical Society, Providence, Rhode Island (1998).

[7] L.C. Evans and W. Gangbo, Differential equations methods for the Monge-Kantorovich mass transfer problem, Mem. Amer. Math. Soc. 137 (1999) 653.

[8] L.C. Evans and R.F. Gariepy, Measure Theory and Fine Properties of Functions, Studies in Advanced Mathematics, CRC Press, Boca Raton, FL (1992).

[9] M. Tsuji, On Lindelöf's theorem in the theory of differential equations. Jap. J. Math. 16 (1939) 149-161. 